\section{Construction of prcK and prcR Mutant Strains of Lactobacillus paracasei HD1.7 and the Impact on the Production of Paracin 1.7}

\author{
Jingping Ge, Xiaolei Ji, Tian You, \\ Yanyang Sun, Wenxiang Ping \\ Key Laboratory of Microbiology, \\ College of Life Science, Heilongjiang \\ University, PR China
}

\begin{abstract}
Gene knockouts of $p r c K, p r c R$ and both together were constructed in L. paracasei HD1.7. The antimicrobial activities of the prcK, prcR and prcKprcR mutant strains against $B$. subtilis were $23.6 \%, 21.9 \%$ and $36.6 \%$ lower than that of the parental strain, respectively, indicating that these genes affect production of bacteriocin antimicrobial peptides. qRT-PCR assays showed that the relative transcription levels of $p r c K$ and $\operatorname{prcR} \mathrm{mRNA}$ in the $\Delta \mathrm{K}$ and $\Delta \mathrm{R}$ strains were 0.36 and 0.33 times of that in parental bacteria, respectively. Our data suggest that $\operatorname{prcK}$ and $\operatorname{prcR}$ are quorum sensing related genes that influence production of the bacteriocin Paracin 1.7. This research provides the basis for exploring the functions of these genes in the production of Paracin 1.7 and more generally for the exploration of the biological preservatives instead of chemical preservatives.
\end{abstract}

\section{Introduction}

Lactobacillus paracasei HD1.7 (CCTCCM 205015) was isolated from Chinese sauerkraut juice in 2003 . In previous studies, the fermentation broth of $L$. paracasei HD1.7 contains a type of peptide, Paracin 1.7, a bacteriocin, that could inhibit the growth of several Gram-positive bacteria $\left(\mathrm{G}^{+}\right)$, Gram-negative bacteria $\left(\mathrm{G}^{-}\right)$and yeast. ${ }^{1}$ The bacterial production process of Paracin 1.7 had characteristics of quorum sensing. Nakayama identified a series of genes in L. paracasei E93490 that were assigned as putative quorum sensing components, and predicted that the signaling molecule of L. paracasei E93490 might have antibacterial activity. ${ }^{2}$ The antibacterial activity may be similar to Paracin 1.7 produced by $L$. paracasei HD1.7.

Quorum sensing in $\mathrm{G}^{+}$is regulated by a two-component regulatory system composed of a histidine protein kinase (HPK) and a phosphor-aspartyl response regulator
(RR). ${ }^{3-5}$ The RR, a DNA binding protein, activates related genes transcription; the phosphorylated RR can bind to the target promoter, directly or indirectly regulating expression of genes. ${ }^{6}$ Quorum sensing in $L$. paracasei is not well understood. Putative histidine protein kinase ( $p r c K)$ and response regulator $(\operatorname{prc} R)$ genes have been identified in L. paracasei E93490 by PCR, but functional studies were not conducted. ${ }^{2}$ Therefore, in this work, we investigated the functions of the $p r c K$ and $p r c R$ genes in quorum sensing and in the potentially related process of the production of antimicrobial peptides.

Gene knockout technology was instrumental in the understanding of quorum sensing. ${ }^{7-10}$ Insertional inactivation has been the main method applied to $\mathrm{G}^{+}$bacteria. ${ }^{11}$ In this method, the flanking sequences of the exogenous DNA imported into host cells and of the target gene in the chromosome of host cells are homologous. The marker gene in the exogenous DNA fragment is therefore inserted into the target gene via homologous recombination, leading to the inactivation of the target gene by the replacement of its DNA.

In this study, we constructed suicide plasmids to create insertional inactivationbased gene knockouts. DNA was incorporated into the chromosome of $L$. paracasei HD1.7 to produce knockouts of $p r c K, p r c R$

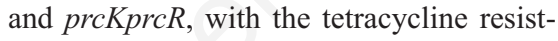
ance gene used as a marker (replacement DNA) in each case. Growth of colonies on plates containing tetracycline indicated that homologous recombination between the suicide plasmids and the host cell had occurred and that knockout mutant strains were produced. Antimicrobial tests were used to show the effects of deletion of $\mathrm{prcK}$ and $p r c R$ on the production of Paracin 1.7, and qRT-PCR was performed to determine whether the expression of $\operatorname{prcK}$ and $\operatorname{prcR}$ mRNA was affected. The data provided the basis for further exploration of the functions of these genes in the production of Paracin 1.7 .

\section{Materials and Methods}

\section{Bacterial strains, plasmids, growth medium and culture conditions}

Bacterial strains and plasmids used in this work are listed in Table 1. L. paracasei HD1.7 strains were propagated in De ManRogosa-Sharpe (MRS) broth (Top Biotech Co., Qingdao, China) or agar at $30^{\circ} \mathrm{C}$ for 24 h. Where appropriate, tetracycline was added to the culture medium at $50 \mu \mathrm{g} / \mathrm{mL}$. E. coli $\mathrm{DH} 5 \alpha$ was grown in Luria-Bertani (LB) broth or agar at $37^{\circ} \mathrm{C}$ with vigorous
Correspondence: Wenxiang Ping, Key Laboratory of Microbiology, College of Life Science, Heilongjiang University, Harbin 150080, PR China.

Fax: +86.0451.86608046.

E-mail: wenxiangp@aliyun.com

Key words: L. paracasei HD1.7, paracin1.7, gene knockout, $\operatorname{prcK}, \operatorname{prcR}$.

Contributions: JG and XJ contributed equally to this work.

Received for publication: 1 November 2017. Revision received: 1 January 2018.

Accepted for publication: 17 January 2018.

This work is licensed under a Creative Commons Attribution NonCommercial 4.0 License (CC BY-NC 4.0).

(C) Copyright J. Ge et al., 2018

Licensee PAGEPress, Italy

Microbiology Research 2018; 9:7475

doi:10.4081/mr.2018.7475

agitation. E. coli $\mathrm{DH} 5 \alpha$ transformant cells harbouring recombinant plasmids were selected onto LB agar plates supplemented with $100 \mu \mathrm{g} / \mathrm{mL}$ (final concentration) of ampicillin, $16 \mathrm{~mL}$ of X-Gal and $4 \mathrm{~mL}$ of IPTG per plate. B.subtilis ATCC 11774 was grown in Beef extract peptone (BP) broth or agar at $37^{\circ} \mathrm{C}$, and this strain was used as an indicator strain when detect the antimicrobial activity.

\section{DNA manipulation and transforma- tion procedures}

Genomic DNA isolation from L. paracasei HD1.7 was performed with TIANGEN genomic tips (Beijing, China). Plasmid isolation from E. coli $\mathrm{DH} 5 \alpha$ transformants was done using TIANGEN plasmid Kit (Beijing, China). For amplication of DNA fragment was used procedure of PCR. The GE system ${ }^{12}$ was used for the reaction system a PCR procedure. For screening purposes, DNA extractions from L. paracasei $\mathrm{HD} 1.7$ and $E$. coli $\mathrm{DH} 5 \alpha$ colonies to be used as the template for PCR were carried out. PCR products were separated by $1 \%$ agar gel electrophoresis and were recovered using the Gel Extraction Kit (Tiangen Biotech CO., Beijing, China). The primers synthesis used in PCR reaction (Table 2) and PCR products sequencing were performed by Invitrogen Corporation.

Electroporation of L. paracasei HD1.7 was also carried out according to the method of Ge. ${ }^{13}$ Transformation of $E$. coli DH5 $\alpha$ competent cells were performed according to the Hannahan method. ${ }^{14}$ Plasmids and restriction digestion products 
were analyzed by agar gel electrophoresis (120V, 20min).

\section{Construction of $p r c K, p r c R$ and prcKprcR knockout mutant of}

\section{L. paracasei HD1.7, respectively}

Restriction enzymes, T4 DNA ligase and DNA-modifying enzymes were used as recommended by the manufacturer (Tiangen, China). To delete the $p r c K, p r c R$ and $\operatorname{prcKprcR}$ from the chromosome of $L$. paracasei HD1.7 by homologous recombination, plasmid $\mathrm{pYTKLKRT,} \mathrm{pYTRLRRT}$ and pYTKRT were constructed (Figure 1).

The pYTKLKRT, a suicide plasmid, which was constructed by inserting a 1400 bp KpnI fragment containing $T e t^{R}$, amplified from pMD18-T-tet using primers Tetup and Tet-down, into the KpnI site of pUC18-KLKR. Plasmid pUC18-KLKR was constructed by inserting a $1370 \mathrm{bp}$ $K p n \mathrm{I} /$ sstI fragment containing $p r c K R$, amplified from pMD18-T-KR using primers prcKR-up and prcKR-down, into the KpnI and PstI sites of plasmid pUC18KL. Plasmid pUC18-KL was constructed by inserting a $1340 \mathrm{bp} \mathrm{SacI/KpnI} \mathrm{fragment}$ containing $p r c K L$, amplified from pMD18T-KL using primers prcKL-up and prcKLdown, into the $S a c \mathrm{I}$ and $K p n \mathrm{I}$ sites of pUC18.

Plasmid pYTRLRRT was constructed by inserting the 1400 bp KpnI fragment containing $\mathrm{Tet}^{R}$ into the KpnI site of pUC18-RLRR. Plasmid pUC18-RLRR was constructed by inserting a 1350 bp KpnI and Pst I fragment containing $p r c R R$ (amplified

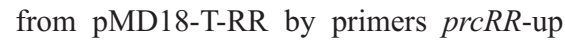
and $p r c R R$-down) into the $K p n \mathrm{I}$ and Pst $\mathrm{I}$ sites of plasmid pUC18-RL. Plasmid pUC18-RL was constructed by inserting a
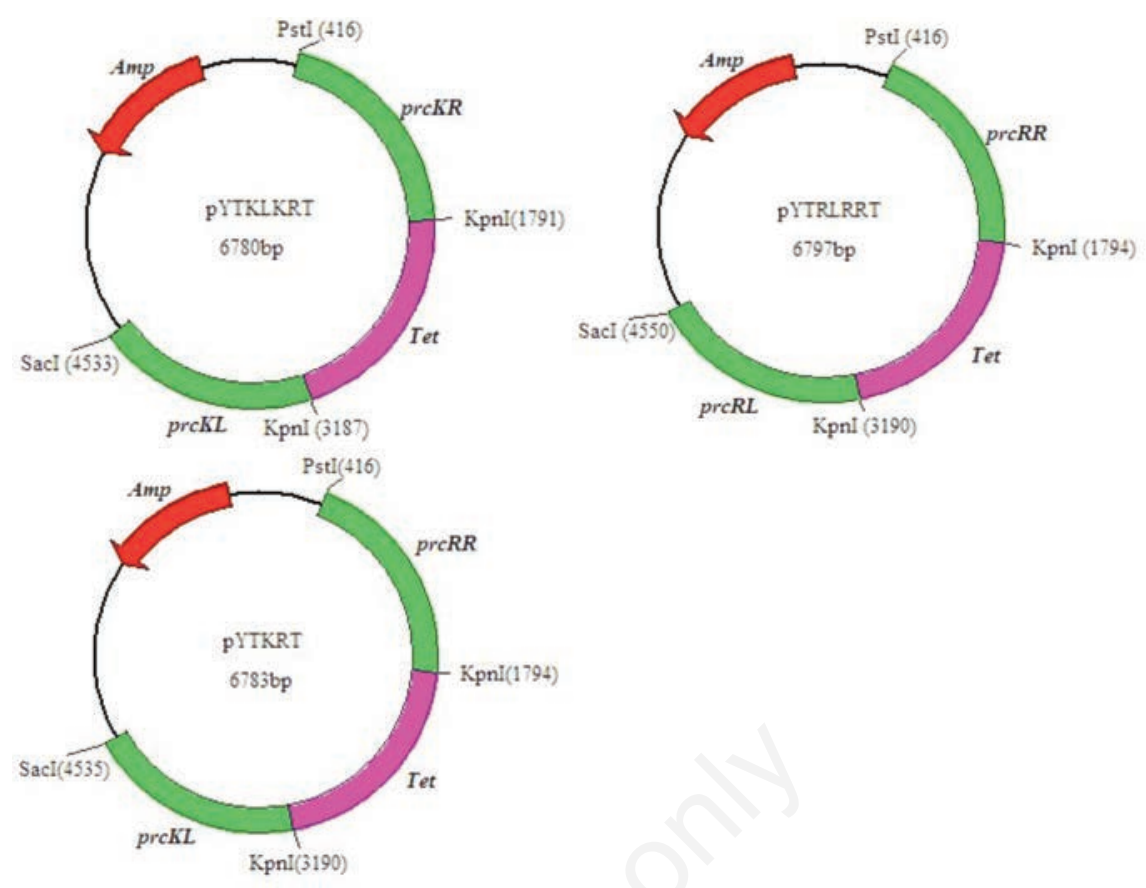

Figure 1. Structures of the pYTKLKRT, pYTRLRRT and pYTKRT plasmids.

Table 1. Strains and plasmids used in this study.

\begin{tabular}{|c|c|c|}
\hline Bacterial strain or plasmid & Relevant features & Reference \\
\hline L. paracasei HD1.7 & Paracin 1.7 producer & Laboratory stock \\
\hline E. coli $\mathrm{DH} 5$ & Host strain for recombinant plasmids & Laboratory stock \\
\hline B. subtlis ATCC 11774 & Indicator strain for bacteriocin activity & Laboratory stock \\
\hline pMD18-T & PCR cloning vector & Invitrogen \\
\hline pYTKLKRT & $\operatorname{prcK} .: \mathrm{Tet}^{R}$ suicide vector & This work \\
\hline pYTRLRRT & $\operatorname{prcR} .:$ Tet $^{R}$ suicide vector & This work \\
\hline pYTKRT & $\operatorname{prcKprcR}:: T^{T} t^{R}$ suicide vector & This work \\
\hline
\end{tabular}

Table 2. Primer sequences used in this study.

\begin{tabular}{|c|c|c|}
\hline Primers & Primer sequences $\left(5^{\prime} \rightarrow 3^{\prime}\right)$ & Targets \\
\hline prcK-up & ATGGAAACTTATTCTGATCTAGCCT & Amplify the whole $\operatorname{prcK}$ gene \\
\hline prcK-down & AAGTCATCTCCCTATAAACAAAGTG & \\
\hline prcKL-up & CCG $\underline{\text { GAGCTC }(S a C I) T A C C T T A A T G A T T T A G A T G C G A G C G ~}$ & Amplify the left homologous arm of $\operatorname{prcK}$ gene, used for construction of pYTKLKRT \\
\hline prcKL-down & GTC $\underline{G T T A C C}($ KpnI)GATTGTTCCTTCGGTGTGGATGTGT & \\
\hline prcKR-up & GTCGGTACC (KpnI)GGTTTTGCCGTCATCAGCGCACTTG & Amplify the right homologous arm of prcK gene, used for construction of pYTKLKRT \\
\hline prcKR-down & CCGCTGCAG(Pst)ACTAATCAGCTGGACTAAGGTGTAT & \\
\hline prcRL-up & CCG & Amplify the left homologous arm of $\operatorname{prc} R$ gene, used for construction of pYTRLRRT \\
\hline prcRL-down & GTC $\underline{G G T A C C(K p n I) A T C T T C A G C A T C G T T T G G T G G T T G G}$ & \\
\hline prcRR-up & GTCGGTACC(KpnI)GGTCAGCATTCGTAGAGTGTCGGCC & Amplify the right homologous arm of $\operatorname{prc} R$ gene, used for construction of pYTRLRRT \\
\hline prcRR-down & CCGCTGCAG(PstI)GCAGTGACCAGAGATAGCTCGGCGT & \\
\hline Amp-up & CTTAGATCT (Bgl II)ACCAATGCTTAATCAGTGAGG & Amplify the ampicillin resistance gene used in pUC18 \\
\hline Amp-down & CGGAGATCT (Bgl II)GGAACCCCTATTTGTTTATTT & \\
\hline Tet-up & CCG $\underline{G G T A C C ~(K p n ~ I) T C T C A T G T T T G A C A G C T T ~}$ & Amplify the tetracycline resistance gene used in pBR322 \\
\hline Tet-down & GTC GGTACC (Kpn I)TAATAGATATGTTCTGCCAAGGGT & \\
\hline prcR-up & ATGACNAAYCAYCARAC & Verify the suicide plasmids pYTRT \\
\hline prcR-down & TGCCAGGTTATGGGAAT & \\
\hline ldh-up & GACACATAAGAAAGGATG & Amplify the whole $l d h$ gene \\
\hline ldh-down & TACTGACGAGTTTCGATGTC & \\
\hline
\end{tabular}


1350 bp SacI/KpnI fragment containing prcRL, amplified from pMD18-T-RL using primers $p r c R L$-up and $p r c R L$-down, into the SacI and KpnI sites of plasmid pUC18.

Plasmid pYTKRT was constructed by inserting the 1400 bp KpnI fragment containing $T e t^{R}$ into the KpnI site of pUC18KLRR. Plasmid pUC18-KLRR was constructed by inserting a 1370 bp KpnI/Pst fragment containing $p r c R R$, amplified from pMD18-T-RR, into the KpnI and PstI sites of plasmid pUC18-KL.

Three recombinant plasmids were transformed by electroporation into L. paracasei HD1.7 cells. The prcK and prcR mutant strains were selected by plating out appropriate dilutions on MRS agar containing 50 $\mu \mathrm{g} / \mathrm{mL}$ (final concentration) of tetracycline. Restriction enzyme analysis and PCR identification were used to investigate whether the suicide plasmids met the experimental design.

\section{Bacteriocin production of the origi- nal and the mutant strains}

To evaluate bacteriocin production, the cultures of the parental and the mutant strains were inoculated into MRS broth and incubated at $37^{\circ} \mathrm{C}$ for $24 \mathrm{~h}$, respectively. The supernatants from cultures were collected for determination of bacteriocin activity using the agar-well diffusion method described by Nwuche. ${ }^{15}$ To eliminate the antimicrobial effect of lactic acid, the $\mathrm{pH}$ of the supernatants were adjusted to 5.5 with $1 \mathrm{M} \mathrm{NaOH}$. Titers were defined as the reciprocal of the highest dilution that inhibited the growth of the indicator strain. The results of the bacteriocin activity assays are presented in arbitrary units per milliliter (AU/mL).

\section{RNA isolation, cDNA synthesis and qRT-PCR}

The cultures of the parental and the mutant strains were inoculated into MRS broth and incubated at $37^{\circ} \mathrm{C}$ for $12 \mathrm{~h}$, respectively. Both these cultures were harvested to extract and purify their RNA. Isolation total RNA was carried out with RNAprep Pure Cell/Bacteria Kit (Tiangen, China) in accordance with the manufacturer's recommendations. RNA concentration was measured at $260 \mathrm{~nm}$ and RNA purity was determined by measuring the absorbance ration at $260 \mathrm{~nm} / 280 \mathrm{~nm}$ with A560 spectrophotometer (AOE INSTRUNMENTS, Shanghai, China).

Reverse transcription was completed using BioRT cDNA First Strand Synthesis Kit (Bioer Technology, China) as instructed. Controls without reverse transcriptase were included in the qRT-PCR runs in order to confirm absence of contaminating DNA.
qRT-PCR amplications were performed with at least 3 replicates using RealMasterMix SYBR Green reagents (Tiangen, China) in a 7500 Real-Time PCR System (Applied Biosystems, Inc., USA). The housekeeping gene $l d h$ was used as internal control L. paracasei HD1.7. The suitability of $l d h$ was verified by isolation of both genomic DNA and RNA during the experiments. Transcriptional levels of $\operatorname{prcK}$ and $\operatorname{prcR}$ were normalized to the transcriptional level of the $l d h$ gene.

\section{Results}

\section{Antimicrobial activity of knockout mutant strains}

Three single colonies growing well on the MRS resistant plates with tetracycline were selected as $p r c K$ knockout mutant strain $(\Delta \mathrm{K}), \operatorname{prc} R$ knockout mutant strain $(\Delta \mathrm{R})$, and $\operatorname{prcKprcR}$ knockout strain $(\Delta \mathrm{KR})$.

The antimicrobial activity of $\Delta K, \Delta \mathrm{R}$ and $\triangle \mathrm{KR}$ were shown in Figure 2. The results showed that the inhibition degrees of $\Delta \mathrm{K}, \Delta \mathrm{R}$ and $\Delta \mathrm{KR}$ were $23.61 \%$ $(1538.29 \pm 46.27 \quad \mathrm{AU} / \mathrm{mL}), \quad 21.93 \%$ $(1572.26 \pm 39.04 \mathrm{AU} / \mathrm{mL})$, and $36.61 \%$ $(1276.53 \pm 21.26 \mathrm{AU} / \mathrm{mL})$ lower than that of original strain $(2013.80 \pm 26.54 \mathrm{AU} / \mathrm{ml})$, respectively, this indicated that the outputs of bacteriocin produced by three mutant

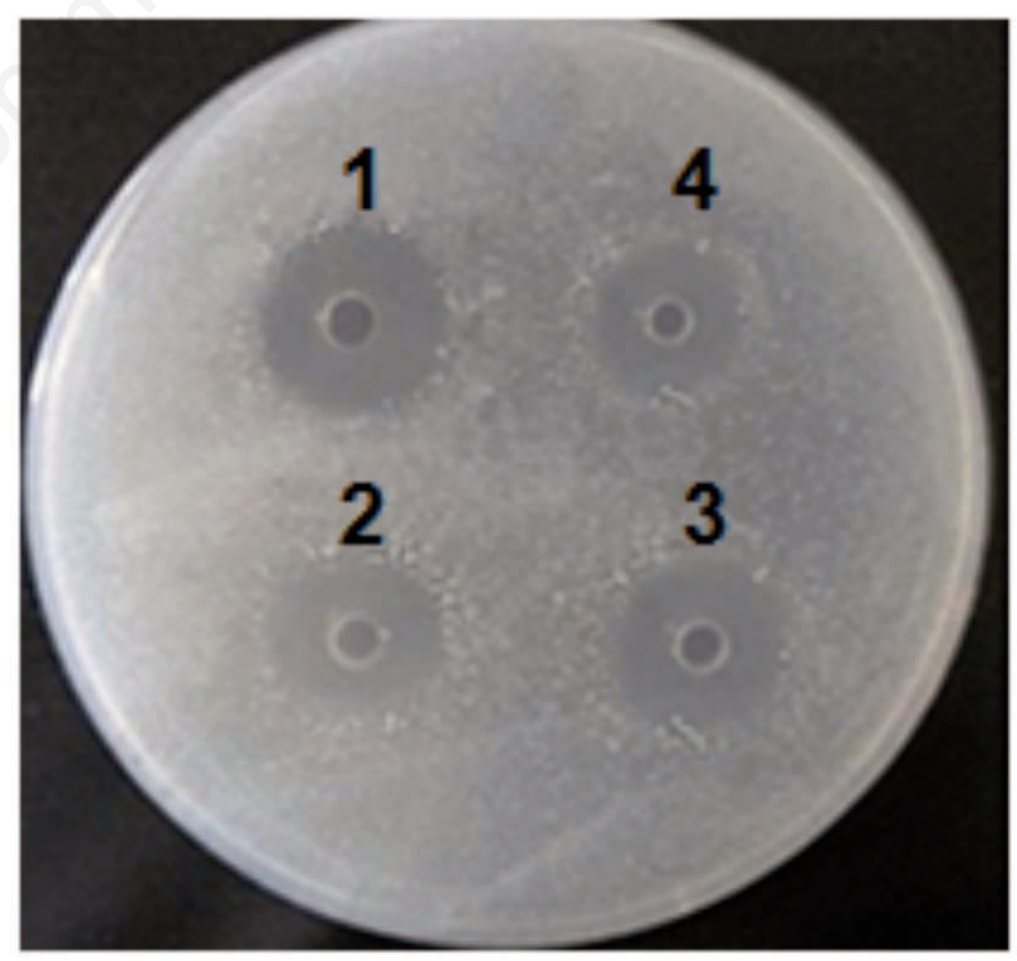

Figure 2. Antibacterial efficacy of mutant and parental $L$. paracase $i$ HD1.7 strains against B. subtilis. 1, 2, 3 and 4 represent the inhibition zones of parental strains, $\triangle K, \triangle R$ and $\triangle \mathrm{KR}$, respectively (pH 5.5). strains were less than that of original strain, and the output of $\Delta \mathrm{KR}$ was obviously less than those of $\Delta \mathrm{K}$ and $\Delta \mathrm{R}$.

However, $\Delta \mathrm{K}$ and $\Delta \mathrm{R}$ still had inhibition ability to the growth of $B$. subtilis. That was probably due to $L$. paracasei HD1.7 having several quorum sensing systems. Other systems would not be impacted if one system was disrupted. Therefore, the mutants still could produce some antimicrobial peptides. Furthermore, the inhibition degree of $\triangle K R$ was $36.6 \%$ lower than that of the wild-type strain, obviously a greater effect than in $\Delta \mathrm{K}$ or $\Delta \mathrm{R}$. Knocking out one gene would negatively influence the regulato a decrease in production of antimicrobial peptides. Knocking out two genes in $\Delta K R$ apparently further increased the negative influence on the regulation of quorum sensing system, leading to a lower production of antimicrobial peptides in $\Delta \mathrm{KR}$ than in $\Delta \mathrm{K}$ or $\Delta \mathrm{R}$.

\section{PCR analysis of $p r c K$ and $p r c R$ knockout mutant strains}

Amplification of both $T e t^{R}$ and ( $p r c K+$ $\mathrm{Tet}^{R}$ ) by PCR was used to identify whether homologous recombination between suicide plasmids and genome of host cells had accomplished, which was based on the theoretical design. tion of the quorum sensing system and lead 
For PCR analysis of $\Delta \mathrm{K}$, genomic DNA of $\Delta \mathrm{K}$ and the primers Tet-up and Tet-down were used as template and the primers, respectively. The original strain L. paracasei HD1.7 was used in the negative control experiment. It was the same with $\Delta \mathrm{R}$ and $\Delta K R$. The results of agarose gel electrophoresis were shown in Figure 3. There was a 1400 bp DNA fragment $\left(T^{R} t^{R}\right)$ that was amplified in $\Delta \mathrm{K}, \Delta \mathrm{R}$, and $\Delta \mathrm{KR}$, but no similar fragment in the negative control experiment, which demonstrated that $T e t^{R}$ was inserted into the genomes of $\Delta \mathrm{K}, \Delta \mathrm{R}$, and $\triangle \mathrm{KR}$.

For PCR identification of $\Delta \mathrm{K}$, genomic DNA of $\Delta \mathrm{K}$ and the primers prcKL-up and

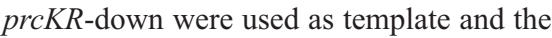
primers, respectively. The original strain $L$. paracasei HD1.7 was used in the negative control experiment. $\Delta \mathrm{R}$ (primers $p r c R L$-up and $p r c R R$-down) and $\Delta \mathrm{KR}$ (primers $p r c K L$-up and $p r c R R$-down) were also identified as described above.

The results of agarose gel electrophoresis were shown in Figure 4. The results of Figure 4A showed that there was a $4140 \mathrm{bp}$ DNA fragment $\left(p r c K+T e t^{R}\right)$ that were amplified in $\Delta \mathrm{K}$ and a $2740 \mathrm{bp}$ fragment (only $p r c K$ ) in the negative control experiment. The results of Figure 4B showed that there was a 4820 bp DNA fragment ( $p r c R+$ $\mathrm{Tet}^{R}$ ) that was amplified in $\Delta \mathrm{R}$ and a 3420 bp fragment (only $p r c R$ ) in the negative control experiment. The results of Figure $4 \mathrm{C}$ showed that there was a 4130 bp DNA fragment $\left(\operatorname{prcKL}+\operatorname{prcRR}+\mathrm{Tet}^{R}\right)$ that was amplified in $\Delta \mathrm{KR}$ and a $3500 \mathrm{bp}$ fragment (only $p r c K+p r c R$ ) in the negative control experiment. It was demonstrated that $T e t^{R}$ was successfully inserted into $\operatorname{prcK}, \operatorname{prcR}$ and $\operatorname{prcKprcR}$, respectively. Therefore, double cross-over occurred in $\Delta \mathrm{K}, \Delta \mathrm{R}$ and $\Delta \mathrm{KR}$. The $\operatorname{prcK}, \operatorname{prcR}$ and $\operatorname{prcKprcR}$ knockout mutant strains were constructed successfully.

\section{qRT-PCR analysis of $p r c K$ and $p r c R$ knockout mutant strains}

Relative transcriptional expression of $\operatorname{prcK}$ and $\operatorname{prcR}$ in the parental and the mutant L. paracasei HD1.7 are present in Figure 5 . There were significant reductions $(p<0.01)$ in $\operatorname{prcK}$ and $p r c R$ transcriptional levels in the parental and the mutant $L$. paracasei HD1.7. The level of prcK mRNA in $\Delta \mathrm{K}$ was $0.36: 1$ compared with the parental strain. For $\Delta \mathrm{R}$, the corresponding ratio was $0.33: 1$. These results indicate that the bacteriocin produced by the mutant $L$. paracasei HD1.7 was indeed mediated by the action of $p r c K$ and $p r c R$ genes. As a consequence, the results of qRT-PCR analysis are consistent with the analysis of PCR and antimicrobial activity.

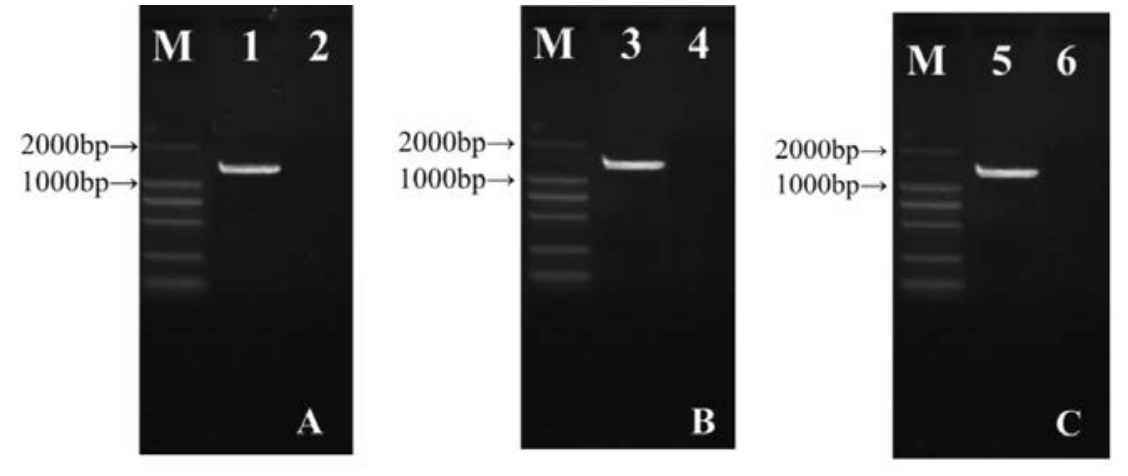

Figure 3. PCR screening results using different templates with Tet-up and Tet-down primers. M: DNA Marker DL 2000; Lane 1, 3, 5: the PCR product using $\triangle K, \triangle R$ and $\triangle K R$ gDNA as template, respectively; Lane 2, 4, 6: the PCR product using the original strain gDNA as template.

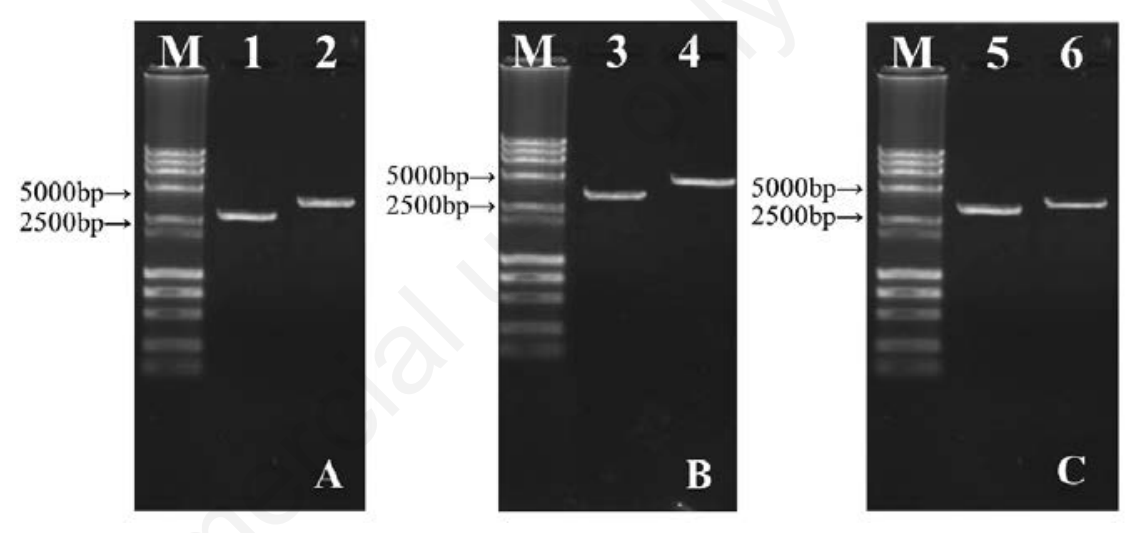

Figure 4. PCR screening for gene knockouts using various primer pairs. (A) The PCR identification result with prcKL-up and prcKR-down primers. (B) The PCR identification result with prcRL-up and prcRR-down primers. (C) The PCR identification result with prcKL-up and prcRR-down primers. M: DNA Marker DL 15000 + 2000; Lane 1, 3, 5: the PCR product using the original strain gDNA as template; Lane 2, 4, 6: the PCR product using $\triangle K, \triangle R$ and $\triangle K R$ gDNA as template, respectively.

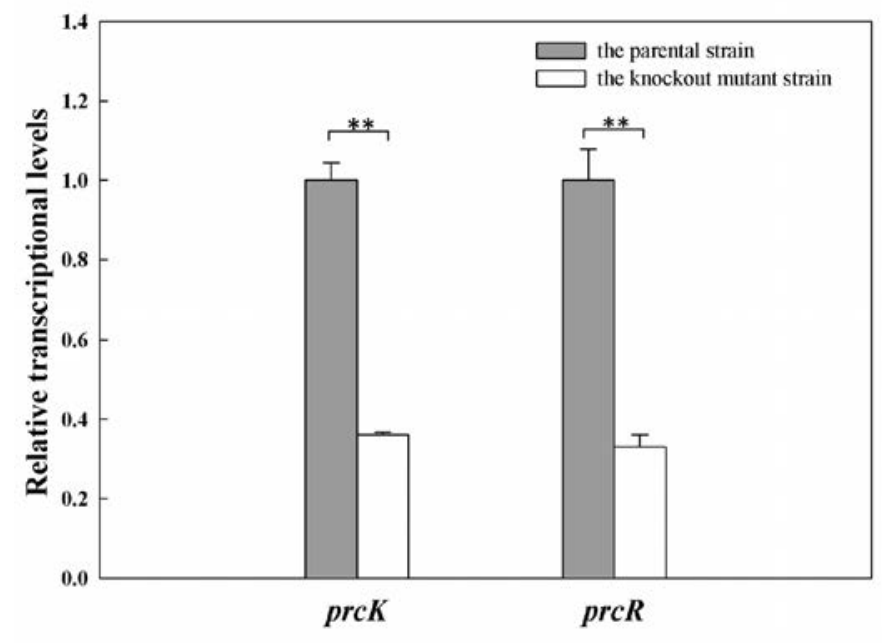

Figure 5. Relative transcriptional expression of prcK and prcR in the parental ( $\bullet$ ) and the mutant $(\square)$ L. paracasei HD1.7. Asterisk indicates a statistically significant difference ( $p$ $<0.01$ ) with respect to the control group. 


\section{Discussion and Conclusions}

Quorum sensing of Gram-positive bacteria is often regulated by three-component regulatory system composed of autoinducing peptide, sensor kinase and response regulator. ${ }^{16}$ When the extracellular AIPs concentration reaches the threshold, specific receptor enzyme proteins can be activated and bind to the histidine protein kinase (HPK) receptors on the cell membrane. The HPK autophosphorylates its own histidine residue and then transfers the phosphate group to the asparitic acid in a response regulator (RR). It has been confirmed that the process of producing bacteriocin from a variety of lactic acid bacteria is regulated by the QS system, such as $L$. sanfranciscensis, ${ }^{17}$ L. acidophilus ${ }^{18,19}$ and L. plantarum. $^{20,21}$

A series of genes mediated quorum sensing and production of Paracin 1.7 by $L$. paracasei HD1.7 has been reported before. Previously, We have demonstrated that the process of producing bacteriocin of L. paracasei HD1.7 is regulated by quorum sensing via the cell density test. ${ }^{13}$ Here, we showed that the inhibition ability of $L$. paracasei HD1.7 decreased after knocking out the $\operatorname{prcK}$ and $\operatorname{prcR}$ genes. This finding suggests that $p r c K$ and $p r c R$ are quorum sensing related genes and influence the production of antimicrobial peptides as $p \ln B$ has been observed for L. paraplantarum L-XM1.22

Different studies have shown the role of $\operatorname{prcK}$ and $\operatorname{prcR}$ in the quorum sensing system. In the study of Nakayama, ${ }^{2}$ the fragment of L. paracasei E93490 amplified by degenerate primers was located in the $p r c K$ gene prior to $p r c R$. Their predictive products PrcK and PrcR are similar to cognate HPK and RR, respectively, and the highest sequence similarity was $L$. sake $\mathrm{SppK}$ and L. plantarum $\mathrm{P} \operatorname{lnB}$, both of which formed a 3CRS that regulates the formation of bacteriocin. PrcK is expected to have six transmembrane alpha-helices at its N-terminal moiety, which can be used as a sensor domain. Similarly, the effect of $p r c K$ and prcR of $L$. paracasei HD1.7 on regulation of bacteriocin production needs further investigation.

In conclusion, the present study shows that the $\operatorname{prcK}$ and $p r c R$ genes of L. paracase $i$ HD1.7 were down-regulated in response to the inhibition ability of $\Delta \mathrm{K}$ and $\Delta \mathrm{R}$. These findings suggested that $\operatorname{prcK}$ and $\operatorname{prcR}$ are quorum sensing related genes and influence the production of antimicrobial peptides. This provides the basis for further exploration of the productions of natural preservatives.

\section{References}

1. Ge JP, Song G, Du CM, et al. Paracin 1 . 7, a bacteriocin produced by Lactobacillus paracasei HD1.7 isolated from Chinese cabbage sauerkraut, a traditional Chinese fermented vegetable food. Acta Microbiol Sinica 2009;49:609-16.

2. Nakayama J, Akkermans AD, De Vos WM. High-throughput PCR Screening of Genes for Three-component Regulatory System Putatively Involved in Quorum Sensing from Low $-\mathrm{G}+\mathrm{C}$ Gram-positive Bacteria. Biosci, Biotech, and Bioc 2003;67:480-9.

3. Desouky SE, Shojima A, Singh RP, et al. Cyclodepsipeptides produced by actinomycetes inhibit cyclic-peptidemediated quorum sensing in Gram-positive bacteria. FEMS Microbiol Lett 2015;362:1-9.

4. Li Z, Nair SK. Quorum sensing: how bacteria can coordinate activity and synchronize their response to external signals?. Protein Sci 2012;21:1403-17.

5. Monnet V, Gardan R. Quorum-sensing regulators in Gram-positive bacteria: 'cherchez le peptide'. Mol Microbiol 2015;97:181-4.

6. Gobbetti M, De AM, Di CR, et al. Cellcell communication in food related bacteria. Int J of Food Microbiol 2007; 120:34-45.

7. Nakashima N, Miyazaki K. Bacterial Cellular Engineering by Genome Editing and Gene Silencing. Int J Mol Sci 2014;15:2773-93.

8. Park JM, Jang YS, Kim TY, et al. Development of a gene knockout system for Ralstonia eutropha H16 based on the broad-host-range vector expressing a mobile group II intron. FEMS Microbiol Lett 2010;309:193-200.

9. Pang X, Liu C, Lyu P, et al. Identification of Quorum Sensing Signal Molecule of Lactobacillus delbrueckii subsp. Bulgaricus. J Agr Food Chem 2016; 28:1835-41.

10. Luong PM, Shogan BD, Zaborin A, et al. Emergence of the P2 phenotype in Pseudomonas aeruginosa PAO1 strains involves various mutations in mexT or mexF. J Bacteriol 2014;196:504-13.

11. Livshits VA, Zakataeva NP, Aleshin $\mathrm{VV}$, et al. Identification and characterization of the new gene rhtA, involved in threonine and homoserine efflux in Escherichia coli. Res Microbiol 2003;154:123-35.
12. Ge JP, Gao XJ, You T, et al. Initial establishment of genetic transformation system for Lactobacillus paracasei HD1.7. Chiese Agricultural Science Bulletin 2011;27:102-8.

13. Ge JP, Fang BZ, Yuan TT, et al. Quorum-sensing behavior of Lactobacillus paracasei HD1.7. Acta Microbiologica Sinica 2011;51:1561-7.

14. Hanahan D. Techniques for transformation of E.coli. DNA cloning 1 1985;109-35.

15. Nwuche CO. Isolation of bacteriocin producing lactic acid bacteria from 'Ugba' and 'Okpiye', two locally fermented nigerian food condiments. Brazilian Archives of Biology $\square$ Technology 2013;56:101-6.

16. Di CR, De AM, Calasso M, et al. Proteomics of the bacterial cross-talk by quorum sensing. J Proteomics 2011;74: 19-34.

17. Di CR, De AM, Limitone A, et al. Cellcell communication in sourdough lactic acid bacteria: A proteomic study inLactobacillus sanfranciscensis CB1. Proteomics 2007;7:2430-46.

18. Moslehijenabian S, Vogensen FK, Jespersen L. The quorum sensing luxS gene is induced in Lactobacillus acidophilus NCFM in response to Listeria monocytogenes. Int J Food Microbiol 2011;149:269-73.

19. Tabasco R, García-Cayuela T, Peláez C, et al. Lactobacillus acidophilus La-5 increases lactacin B production when it senses live target bacteria. Int $\mathrm{J}$ Food Microbiol 2009;132:109-16.

20. Man LL, Meng XX, Zhao RH. Induction of plantaricin MG under coculture with certain lactic acid bacterial strains and identification of LuxS mediated quorum sensing system in Lactobacillus plantarum KLDS1.0391. Food Control 2012;23:462-9.

21. Sturme MHJ, Francke C, Siezen RJ, et al. Making sense of quorum sensing in lactobacilli: a special focus on Lactobacillus plantarum WCFS1. Microbiology 2007;153:3939-47.

22. Zhang XM, Shang N, Zhang X, et al. Role of plnB gene in the regulation of bacteriocin production in Lactobacillus paraplantarum L-XM1. Microbiol Res 2013;168:305-10. 